\title{
Dynamic and Probabilistic Relation between Meteoroids and their Parent Bodies
}

\author{
Lars G. Adolfsson and Bo A. S. Gustafson \\ Department of Astronomy, PO Box 112055, University of Florida, \\ Gainesville, Florida 32611-2055, USA
}

\begin{abstract}
We estimate the probability that specific meteoroids were produced by a specific parent body and obtain the ejection velocity and several other conditions of the ejection as a by-product. The duration of cometary activity leading to a meteor stream, or the epoch of a collision can be estimated from the ejection times of meteoroids in the same stream. The level of activity of a comet or probability of asteroid collisions can be estimated from the part of the orbit where ejection took place. We show that Phaethon ejected Geminid meteoroids over at least 3000 years lasting to as recently as $\approx \mathrm{AD} 1600$. Ejections are predominantly in the direction of the sun, i.e., from Phaethon's day side, and give insights into the ejection process which is suggestive of cometary activity. Our calculations account for the uncertainty in the observational data and include the effects of planetary perturbations, radiation pressure, Poynting-Robertson light drag and solar wind corpuscular drag.
\end{abstract}

\section{Parent-Daughter Association}

The trajectory of an ejected meteoroid separates from that of the parent due to differences in forces acting on the parent and on the meteoroid, and due to the velocity difference acquired in the ejection process. While the trajectories may intersect by chance, they usually do so at high relative velocities reflecting the difference in the orbits. The meteoroid and the parent body therefore share only one point in space where their relative velocity is low; the point of ejection. We apply a refined version of Gustafson's (1989a) method to identify this point and extract the conditions of ejection of 43 Prairie Network (PN) Geminids, eight PN Taurids and four PN $\alpha$ Capriconids from their proposed parent bodies: 3200 Phaethon, $\mathrm{P} /$ Encke and $\mathrm{P} /$ Honda-Mrkos-Pajdušákova (P/H-M-P) respectively.

The first step is to estimate the error in the velocity vector assigned to each meteor observation. The orbit probability distribution for any epoch is probed using the error estimate and numerical integrations. These distributions include uncertainties in the numerical integrations and account for any passage through chaotic orbital regions. Similar probing can be used to account for the uncertainty on the parent body's trajectory. A comparison of the two distributions reveals the probability of transfer from the parent as a function of time. It also gives a probability for the required energy or ejection velocity vector. 
Uncertainties in the orbital period are so large that the position of a meteoroid along the orbit can typically be considered to be unknown except during the last few orbits before atmospheric entry (Gustafson, 1989a). We therefore do not search for a time and point in space where the meteoroid's location coincides with the location of the presumed parent. Following Gustafson, we use the next best dynamic criterion for a genetic relation; orbital intersections and take advantage of the fact that points of orbital intersection must be along the line of nodes. One node from each orbit coincides whenever there is an orbital intersection. Numerical integrations yield the orbit of the meteoroid and the parent at discrete times. We take advantage of the fact that the nodes move in a continuous manner along the line of nodes. Whenever a node shifts past another node, there must be at least one orbital intersection in that time step.

The ejection velocity vector is simply the vector difference of the orbital velocities at the moment of ejection. A point is rejected as a potential ejection site unless the relative velocity is deemed to be a physically realistic ejection velocity. We use the gas velocity as an upper limit to the ejection velocity during cometary activity. Gustafson (1989b) showed that even massive particles may approach the gas velocity if they are pieces of a thin mantle. We estimate the gas velocity from the empirical relation $0.58 d^{-0.5} \mathrm{~km} \mathrm{sec}^{-1}$, where $d$ (AU) is the heliocentric distance (Delsemme, 1982).

Uncertainties in all starting parameters propagate through the orbital integrations and produce a distribution of orbits for each meteoroid and each epoch. Uncertainties in the orbit of the parent body are much smaller than the corresponding uncertainties for the meteoroids so that only the central orbit of the parent body is used in this article. We estimate the probability of ejection in a time interval from the fraction of the distribution that shifted from one side of the parent's node to the other. We also obtain the epoch of ejection, the ejection velocity vector (direction and magnitude), the position along the orbit at the time of ejection and the probability of ejection at points that satisfy all of the conditions for ejection. It is possible to estimate the duration of the formation process of the sampled part of a meteor stream from the ejection times of meteoroids belonging to the same stream.

We need the position, pre-atmospheric velocity and cross-section to mass ratio of each meteoroid to integrate their orbits back in time. Apart from gravitational forces due to the Sun and all major planets except Pluto, we also account for radiation pressure (as soon as the meteoroid leaves the Earth's shadow), Poynting-Robertson (P-R) light drag and corpuscular solar wind drag. The corpuscular drag is $30 \%$ of $\mathrm{P}-\mathrm{R}$ drag, which is the approximate value during nominal solar wind conditions (Gustafson, 1994). All non-gravitational forces are estimated from the cross-section to mass ratio of the meteoroid obtained from the atmospheric trajectory (Gustafson \& Adolfsson, 1996).

We use only the most precise meteor observations available that have been successfully interpreted through single-body theory (Pecina \& Ceplecha 1983). Single-body theory provides error estimates for the velocity and the cross-section to mass ratio directly as part of the solution. We use stream fireball data from the Prairie Meteorite Network program (McCrosky et al., 1978; 1979). These data have been re-reduced by Ceplecha and kindly made available to us. Table 1 lists pertinent data for the fireballs and their potential parent bodies. The column labeled "N" denotes the number of sample trajectories per meteoroid. 
The combination of a small cross-section to mass ratio for fireballs, and short integration times $\left(\approx 10^{4} \mathrm{yr}\right)$, implies that the non-gravitational effects are negligible with respect to the errors in the fireball data. However, for completeness, they are included in the integrations.

Table 1. Proposed parent bodies and PN stream fireballs

\begin{tabular}{llrrr} 
Parent body & Stream & mass $(\mathrm{g})$ & $\mathrm{N}$ & End epoch \\
\hline 3200 Phaethon & 43 Geminids & $10-2 \times 10^{3}$ & 26 & $-5,000$ \\
1566 Talos & & & & \\
5786 Icarus & & & & \\
\hline P/Encke & 8 Taurids & $90-400$ & 6 & $-20,000$ \\
\hline $\begin{array}{l}\text { P/Honda-Mrkos-Pajdušákova } \\
\text { 2101 Adonis }\end{array}$ & $4 \alpha$ Capriconids & $10-700$ & 6 & $-20,000$ \\
& & & &
\end{tabular}

\section{Numerical Results And Conclusions}

All Taurids had at least one orbital intersection with $\mathrm{P} /$ Encke, but the relative velocity is 1.9 to 16 times higher than the estimated gas velocity, implying that no genetic relation is present. However, it should be noted, that only six orbits were sampled from the error distributions for each Taurid and that P/Encke is known to have non-gravitational forces. These issues should be addressed before a definite statement is made about the direct formation or the possibility of indirect formation of Taurids by way of intermediary bodies in a larger complex (see Asher et al. 1993). All four $\alpha$ Capriconids have orbital intersections with $\mathrm{P} / \mathrm{H}-\mathrm{M}-\mathrm{P}$ and three with 2101 Adonis, but the relative velocities are over 6 times the estimated gas velocity. P/H-M-P had close encounters with Jupiter in the past due to its low inclination $\left(4^{\circ}\right)$ and aphelion distance (5.5 AU). A distribution of orbits should be used for the parent in a more complete work, rather than just the central orbit used here.

All but one of the Geminids have orbital intersections with Phaethon. Twenty-three of the 43 Geminids satisfy all criteria for a parent-daughter association. We also searched for a relationship to 1566 Icarus and 5786 Talos. No intersections were found between the Geminids and 1566 Icarus. All Geminids intersected the orbit of 5786 Talos, but the relative velocities exceed $50 \mathrm{~km}$ $\mathrm{sec}^{-1}$, which is far above any reasonable ejection velocity.

Figure 1 illustrates where in the orbit and with what direction the $23 \mathrm{Gem}$ inids may have escaped from Phaethon. The figure also shows the probability of ejection as a function of time. This confirms Gustafson's (1989a) conclusions that: (1) The Geminids can be dynamically related to Phaethon. (2) The distribution of ejection velocity vectors is skewed (18 out of 23 vectors) toward the Sun so that ejection was from Phaethon's day side. The improved reduction to orbital data and the probabilistic treatment allow us to conclude that Phaethon was active over at least 3000 years up to quite recently $(\approx A D 1600)$. Phaethon was once an active comet and cometary activity created the Geminid meteoroid stream.

Note that although we account for random errors in the meteor orbit determinations, we cannot account for unknown systematic errors that may still 

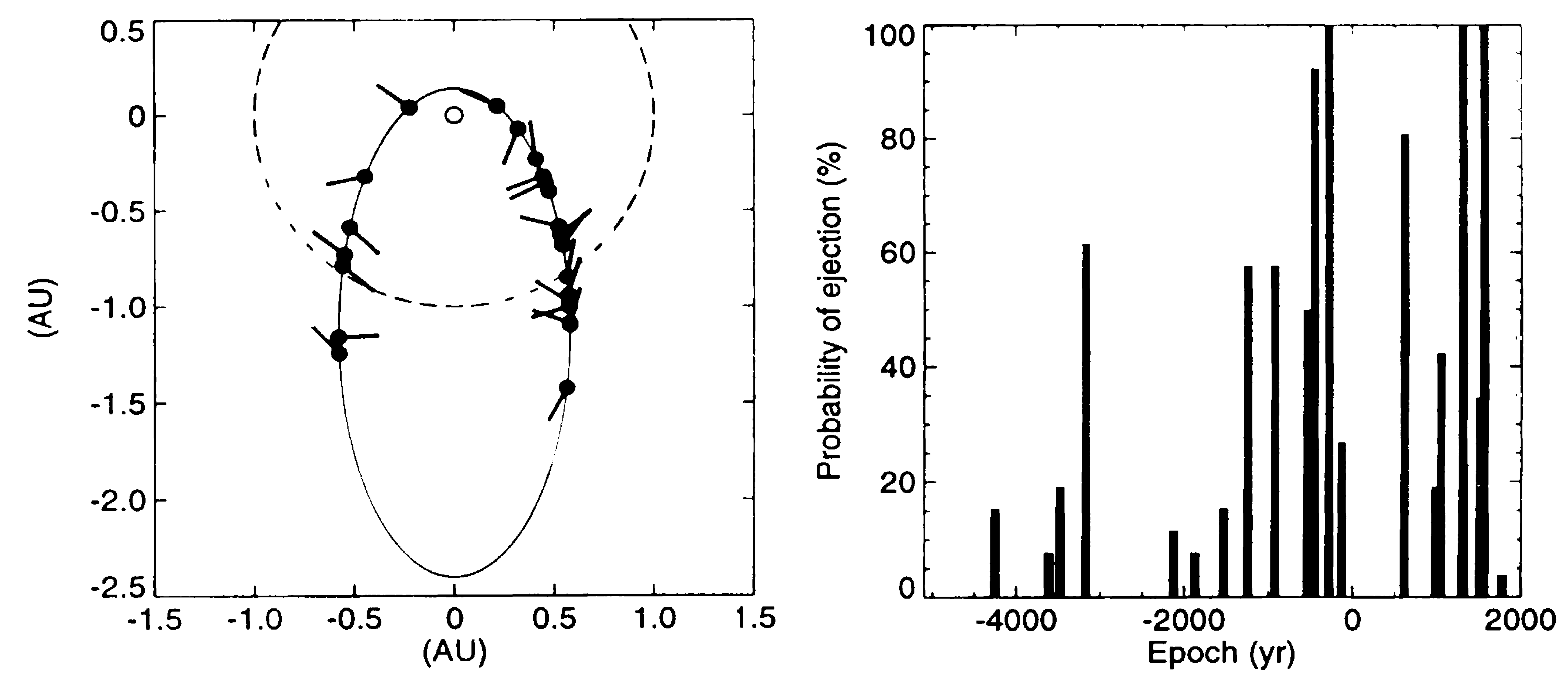

Figure 1. Left: Position and direction of ejection for the 23 Geminids. Right: Probability of ejection for the 23 Geminids as a function of time.

plague the observations or may be introduced in the reduction of the data. The true ejection point is most certainly not found because of the finite sampling of the probability distribution of the orbits. Even the correct epoch may be missed if the integrations are not carried sufficiently far back in time. Criteria may be developed for the minimum integration time for stream meteoroids but computer cycle availability was the real limitation. Also note that any reaction force on meteoroids from outgassing is not included. Similarly, all forces acting on the parent may not be accounted for, i.e., the non-gravitational force due to asymmetric outgassing.

Acknowledgments. We are grateful to Prof. Zdeněk Ceplecha for useful discussions on atmospheric entry and for the use of unpublished single-body solutions for the PN stream fireballs. This work was supported in part by the NASA Planetary Geology and Geophysics Program and by the Division of Sponsored Research of the University of Florida.

\section{References}

Asher, D.J., S.V.M. Clube, and D. I. Steel. 1993, MNRAS, 264, 93

Delsemme, A.H. 1982, in Comets, L.L. Wilkening, Tucson: U Az. Press, 85

Gustafson, B. A. S. 1989a, A\&A, 225, 533

Gustafson, B. A. S. 1989b, ApJ, 337, 945

Gustafson, B. Å. S. 1994, Annu. Rev Earth Planet. Sci., 22, 553

Gustafson, B. A. S., \& L.G. Adolfsson. 1996, Earth, Moon and Planets, in press.

McCrosky, R.E., C.-Y Shao, and A. Posen. 1978, Meteoritika, 37, 44

McCrosky, R.E., C.-Y. Shao, and A. Posen. 1979, Meteoritika, 38, 106

Pecina, P., \& Z. Ceplecha. 1983, Bull. Astron. Inst. Czechosl., 35, 120 